\title{
Small Interfering RNA-Mediated Suppression of Fas Modulate Apoptosis and Proliferation in Rat Intervertebral Disc Cells
}

\author{
Jong-Beom Park, Chanjoo Park \\ Department of Orthopaedic Surgery, Uijeongbu St. Mary's Hospital, College of Medicine, The Catholic University of Korea, Seoul, Korea
}

\begin{abstract}
Study Design: In vitro cell culture model.
Purpose: To investigate the effect of small interfering RNA (siRNA) on Fas expression, apoptosis, and proliferation in serum-deprived rat disc cells.

Overview of Literature: Synthetic siRNA can trigger an RNA interference (RNAi) response in mammalian cells and precipitate the inhibition of specific gene expression. However, the potential utility of siRNA technology in downregulation of specific genes associated with disc cell apoptosis remains unclear.

Methods: Rat disc cells were isolated and cultured in the presence of either $10 \%$ fetal bovine serum (FBS) (normal control) or $0 \%$ FBS (serum deprivation to induce apoptosis) for 48 hours. Fas expression, apoptosis, and proliferation were determined. Additionally, siRNA oligonucleotides against Fas (Fas siRNA) were transfected into rat disc cells to suppress Fas expression. Changes in Fas expression were assessed by reverse transcription-polymerase chain reaction and semiquantitatively analyzed using densitometry. The effect of Fas siRNA on apoptosis and proliferation of rat disc cells were also determined. Negative siRNA and transfection agent alone (Mock) were used as controls.

Results: Serum deprivation increased apoptosis by $40.3 \%$ ( $p<0.001)$, decreased proliferation by $45.3 \%(p<0.001)$, and upregulated Fas expression. Additionally, Fas siRNA suppressed Fas expression in serum-deprived cultures, with $68.5 \%$ reduction at the mRNA level compared to the control cultures $(p<0.001)$. Finally, Fas siRNA-mediated suppression of Fas expression significantly inhibited apoptosis by $9.3 \%$ and increased proliferation by $21 \%$ in serum-deprived cultures ( $p<0.05$ for both).

Conclusions: The observed dual positive effect of Fas siRNA might be a powerful therapeutic approach for disc degeneration by suppression of harmful gene expression.
\end{abstract}

Keywords: Disc cells; Fas; Apoptosis; Cell proliferation; Small interfering RNA

\section{Introduction}

RNA interference (RNAi) facilitates inhibition of specific genes by sequence-specific gene silencing using doublestranded RNA (dsRNA) [1,2]. This approach involves post-transcriptional gene silencing via dsRNA-mediated inhibition of gene expression through degradation of a specific, targeted mRNA. Small interfering RNA (siRNA), a type of RNAi, is a hybrid consisting of a sense and an antisense strand homologous in sequence to the sup-

Received Nov 9, 2016; Revised Dec 11, 2016; Accepted Dec 13, 2016

Corresponding author: Jong-Beom Park

Department of Orthopaedic Surgery, Uijeongbu St. Mary’s Hospital, College of Medicine, The Catholic University of Korea, 271 Cheonbo-ro, Uijeongbu 11765, Korea

Tel: +82-31-820-3578, Fax: +82-31-847-3671, E-mail: spinepjb@catholic.ac.kr 
pressed gene [3]. Therefore, synthetic siRNA can trigger an RNAi response in mammalian cells and induce inhibition of specific gene expression. The specificity and potency of synthetic siRNA facilitate elucidation of gene function and investigate novel approaches for the treatment of various diseases [4]. However, the potential utility of siRNA technology in downregulation of specific genes associated with disc cell apoptosis remains unclear.

Fas is a type-I $45-\mathrm{kDa}$ transmembrane protein that belongs to the tumor necrosis factor family. A wide range of human cells express Fas on their plasma membrane $[5,6]$. After binding of its natural ligand (Fas ligand), Fas triggers apoptosis in susceptible cells via two major downstream pathways: caspase 9-mediated intrinsic pathway and caspase 8-mediated extrinsic pathway. Previous studies reported a higher rate of apoptosis due to Fas/Fas ligand-mediated caspase activation in degenerated or herniated discs [7-9]. In addition, the extent of Fas expression was correlated with the severity of disc degeneration [7]. Therefore, specific downregulation of Fas might represent a novel therapeutic strategy against disc degeneration due to inappropriate apoptosis of disc cells.

In the current study, we investigated the effect of siRNA-mediated suppression of Fas on apoptosis and proliferation in rat disc cells, by transfection of Fas-targeting siRNA into cells for 48 hours under conditions of serum deprivation.

\section{Materials and Methods}

The experimental methods of the current study were the same as described in the previous paper [10].

\section{Intervertebral disc cell culture}

The Animal Care and Use Committee of the author's institution approved all experiments. Lumbar intervertebral discs (L1-L6) were harvested from five 4-week-old male Sprague Dawley rats immediately after sacrifice. The discs were carefully dissected under a microscope to isolate nucleus pulposus tissues, which were cultured in Dulbecco's modified Eagle's medium (DMEM; Gibco BRL, Grand Island, NY, USA) supplemented with $10 \%$ fetal bovine serum (FBS; Hyclone, Ottawa, Ontario, Canada), 100 U/ $\mathrm{mL}$ penicillin (Gibco BRL), and $100 \mathrm{mg} / \mathrm{mL}$ streptomycin (Gibco BRL) in a humidified $5 \% \mathrm{CO}_{2}$ incubator at $37^{\circ} \mathrm{C}$ for 12 hours. To isolate disc cells, nucleus pulposus tis- sues in DMEM medium were digested with $0.2 \%$ pronase (Sigma Chemical, St. Louis, MO, USA) for 4 hours. After enzymatic digestion, the suspension was filtered through a $70-\mu \mathrm{m}$ mesh (Falcon, Franklin Lakes, NJ, USA). Filtered cells were then washed with DMEM and grown as primary cultures. After seven passages, rat disc cells were trypsinized, subcultured into six-well plates at $1 \times 106$ cells/well, and cultured in the presence of either 10\% FBS (normal control) or 0\% FBS (to induce apoptosis) for 48 hours.

\section{Terminal deoxynucleotidyl transferase-mediated dUTP nick-end labeling assay}

Apoptosis of disc cells was determined using a terminal deoxynucleotidyl transferase-mediated dUTP nick-end labeling (TUNEL) assay. Briefly, $15 \mu \mathrm{L}$ APOPercentage dye (Biocolor Life Science, Carrickfergus, UK) was added to culture media to stain cells for 30 minutes. After removal of the culture medium containing the dye, the cells were gently washed twice with $500 \mu \mathrm{L} /$ well phosphate-buffered saline (PBS) and transferred to an inverted microscope to capture images. Disc cells cultured for 48 hours were used as controls.

\section{Flow cytometry}

Apoptosis of disc cells was also determined by double staining with fluorescein isothiocyanate (FITC)-conjugated Annexin V and propidium iodide (PI) (Pharmingen, San Diego, CA, USA) according to the manufacturer's instructions. Briefly, cells were washed with cold PBS and suspended in binding buffer $(10 \mathrm{mM}$ HEPES-NaOH $\mathrm{pH}$ 7.4, $140 \mathrm{mM} \mathrm{NaCl}, 2.5 \mathrm{mM} \mathrm{CaCl}_{2}$ ) at $1 \times 10^{6}$ cells/well and stained with $5 \mu \mathrm{L}$ Annexin V-FITC and $5 \mu \mathrm{L}$ PI. Cells were analyzed using a FACScan flow cytometer (Becton Dickinson, San Jose, CA, USA) at 48 hours.

\section{Cell proliferation assay}

Proliferation of disc cells was determined by the colorimetric CellTiter 96 AQueous One Solution cell proliferation assay (Promega, Madison, WI, USA). Briefly, disc cell cultures in media supplement with $10 \%$ FBS or $0 \%$ FBS were added to the wells of a 96-well plate, and the medium was allowed to equilibrate for 1 hour. Next, $20 \mu \mathrm{L}$ 3-(4,5-dimethylthiazol-2-yl)-5-(3- 
carboxymethoxyphenyl)-2-(4-sulfophenyl)-2H-tetrazolium, inner salt (MTS), and phenazine methosulfate were added to each well. After incubation for 2 hours at $37^{\circ} \mathrm{C}$ in a humidified $5 \% \mathrm{CO}_{2}$ incubator, absorbance of the plate at $490 \mathrm{~nm}$ was recorded using a spectrophotometric plate reader. Data are presented as means \pm standard deviation from six replicates.

\section{Western blotting}

Fas protein levels in disc cells were determined by Western blot analysis according to the manufacturer's instructions (Santa Cruz Biotechnology, Paso Robles, CA, USA). Briefly, disc cells were washed with ice-cold PBS and lysed with protein lysis buffer containing $50 \mathrm{mM}$ HEPES $\mathrm{pH}$ 7.5, $150 \mathrm{mM} \mathrm{NaCl}, 1.5 \mathrm{mM} \mathrm{MgCl} 2,1 \mathrm{mM}$ EGTA, $10 \%$ glycerol, $1 \%$ Triton X-100, and $1 \mu \mathrm{M}$ phenylmethylsulphonyl fluoride. Cell lysates were centrifuged at 12,000 g for 15 minutes, and protein concentrations were measured using the bicinchoninic acid method (Thermo Fisher Scientific, Pittsburgh, PA, USA). A total of $50 \mu$ g protein per sample was electrophoresed on $10 \%$ sodium dodecyl sulfatepolyacrylamide gels and transferred onto nitrocellulose membranes. The membranes were incubated with a primary antibody against Fas (Santa Cruz Biotechnology), followed by incubation with a horseradish peroxidaselinked IgG secondary antibody (BioRad, Richmond, CA, USA). Immunoreactive bands were visualized using an enhanced chemiluminescence detection kit (Santa Cruz Biotechnology).

\section{Transfection with siRNA against Fas}

Disc cells were grown to reach $80 \%-85 \%$ confluency on the day of transfection. GeneBank accession no. for Fas was NM139194. The following siRNA constructs were used in this study: Fas siRNA sense, 5' -GAAACGAACUGCACCCGGAU-3'; negative siRNA sense, 5' -UAGCGACUAAACACAUCAA-3' (Dharmacon,
Thermo Fisher Scientific). Briefly, cells were transfected with siRNA using the DharmaFECT siRNA transfection reagent (Dharmacon) according to the manufacturer's instructions. Transfections were performed with serum-free a-modified Eagle's medium containing $200 \mathrm{nmol}$ siRNA and $6 \mu \mathrm{L}$ DharmaFECT. After 18 hours, the transfection medium was replaced with a complete medium, and the cells were collected at 48 hours for analysis of Fas silencing by reverse transcription polymerase chain reaction (RT-PCR).

\section{Reverse transcription polymerase chain reaction}

Total RNA from disc cells was extracted using TRIzol reagent (Invitrogen, Grand Island, NY, USA) according to the manufacturer's instructions. For cDNA synthesis, $2 \mu \mathrm{g}$ total RNA was reverse transcribed in a reaction mixture containing 25 units of ribonuclease inhibitor, 15 units of reverse transcriptase, $500 \mathrm{ng}$ Oligo (dT) primers, $3 \mathrm{mM}$ $\mathrm{MgCl}_{2}, 0.5 \mathrm{~mm} \mathrm{dNTP}$, and $1 \times \mathrm{RT}$ buffer (Promega). Synthesized cDNA was used as a template for polymerase chain reaction (PCR) amplification with GoTaq polymerase (Promega) and primers for Fas (Bioneer, Cheongwon, Korea). PCR was performed with the Mycycler thermal cycler (BioRad, Hercules, CA, USA) according to the manufacturer's instructions. Analysis of the PCR products in $1 \%$ agarose gels showed single-band amplification products of expected sizes. Primer sequences and experimental conditions of PCR are summarized in Tables 1 and 2. Blots were semiquantitatively analyzed using

Table 1. The primer sequences of polymerase chain reaction

\begin{tabular}{|c|c|c|}
\hline Primer & Sequence & Size (bp) \\
\hline \multirow[t]{2}{*}{ Fas } & 5'-GATGTGGAACTGGCAGAG-3' & 487 \\
\hline & 5'-GGCTGACTTTCTCCTGGT-3' & \\
\hline \multirow[t]{2}{*}{ GAPDH } & 5'-ATCATCTCCGCCCCTTCTGC-3' & 437 \\
\hline & 5'-GCCTGCTTCACCACCTTCTT-3' & \\
\hline
\end{tabular}

GAPDH, glyceraldehyde-3-phosphate dehydrogenase.

Table 2. The experimental conditions of polymerase chain reaction

\begin{tabular}{|c|c|c|c|c|}
\hline \multirow{2}{*}{ Primer } & \multicolumn{3}{|c|}{ Experimental conditions } & \multirow{2}{*}{ Cycle } \\
\hline & Denaturation & Annealing & Polymerization & \\
\hline Fas & $94^{\circ} \mathrm{C} 5 \mathrm{~min}$ & $94^{\circ} \mathrm{C} 30 \mathrm{sec} \rightarrow 46.3^{\circ} \mathrm{C} 30 \mathrm{sec} \rightarrow 72^{\circ} \mathrm{C} 30 \mathrm{sec}$ & $72^{\circ} \mathrm{C} 5 \mathrm{~min}$ & 35 \\
\hline GAPDH & $94^{\circ} \mathrm{C} 5 \mathrm{~min}$ & $94^{\circ} \mathrm{C} 30 \mathrm{sec} \rightarrow 56^{\circ} \mathrm{C} 30 \mathrm{sec} \rightarrow 72^{\circ} \mathrm{C} 30 \mathrm{sec}$ & $72^{\circ} \mathrm{C} 5 \mathrm{~min}$ & 25 \\
\hline
\end{tabular}

GAPDH, glyceraldehyde-3-phosphate dehydrogenase. 

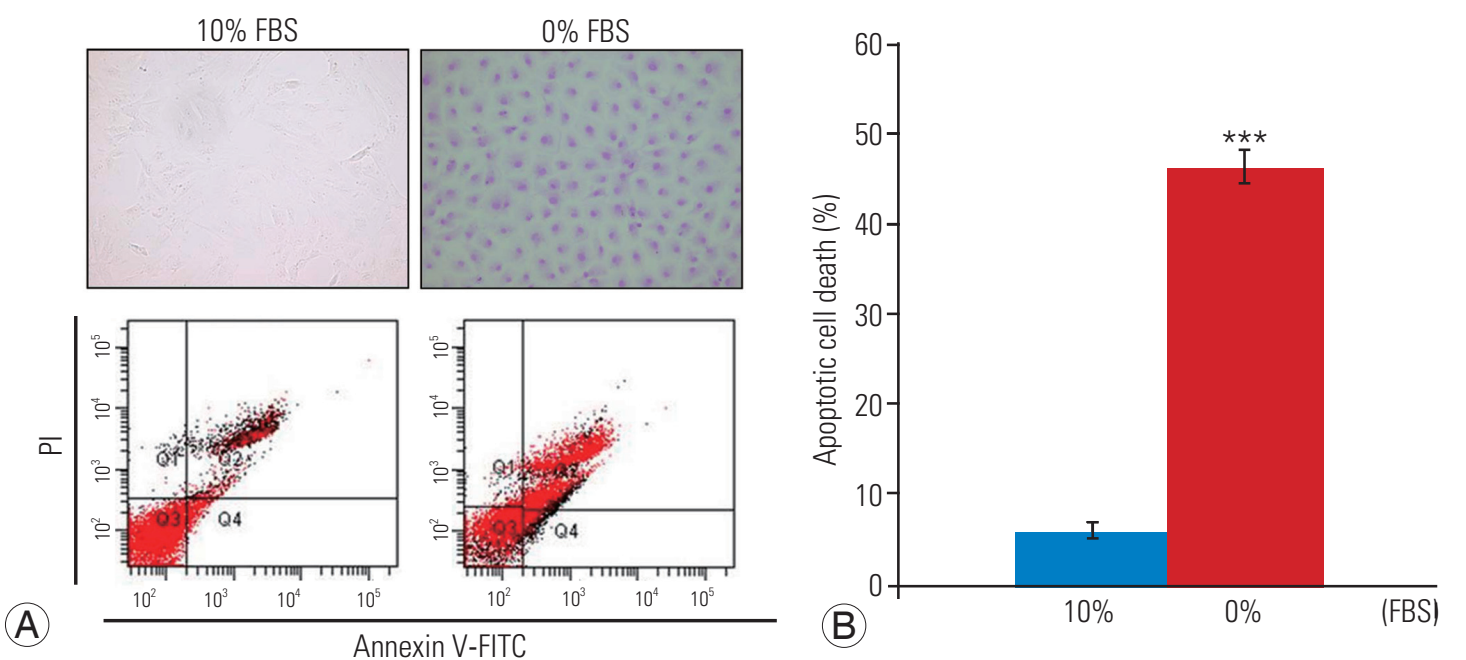

Fig. 1. (A) Terminal deoxynucleotidyl transferase-mediated dUTP nick-end labeling and flow cytometric analysis. PI, propidium iodide; V-FITC, Fluorescein isothiocyanate-conjugated Annexin V. (B) Disc cells incubated in 0\% fetal bovine serum (FBS) for 48 hours displayed a significantly greater rate $40.3 \%$ for apoptotic cell death as compared to cells incubated in $10 \%$ FBS $(5.9 \% \pm 1.1 \%$ vs. $46.2 \% \pm 3.5 \%$ ). ${ }^{* * *} p<0.001$.

GF670 imaging densitometer and the Molecular Analyst software (Bio-Rad), and results were expressed relative to the expression of Gapdh housekeeping gene. All experiments were repeated three times per sample, and average of three technical replicates was used as the final band density.

\section{Effect of siRNA-mediated suppression of Fas on apop- tosis and proliferation}

TUNEL, flow cytometry, and MTS assay were used to investigate the effect of siRNA-mediated suppression of Fas expression on apoptosis and proliferation of disc cells using the methods described above. Statistical analyses were performed using Student's $t$-test. A $p$-values of $<0.05$ were considered to indicate statistical significance.

\section{Results}

\section{Serum deprivation induces Fas upregulation and apoptosis and inhibits proliferation of disc cells}

TUNEL assay and flow cytometry results are presented in Fig. 1A. There was a significant increase in apoptotic death in disc cells incubated in a serum-deprived medium than in those incubated in a medium containing $10 \%$ FBS for 48 hours $(46.2 \% \pm 3.5 \%$ vs. $5.9 \% \pm 1.1 \%, p<0.001)$ (Fig. $1 \mathrm{~B}$ ). Similarly, the MTS assay revealed that the proliferation rate of disc cells cultured in the serum-deprived medium

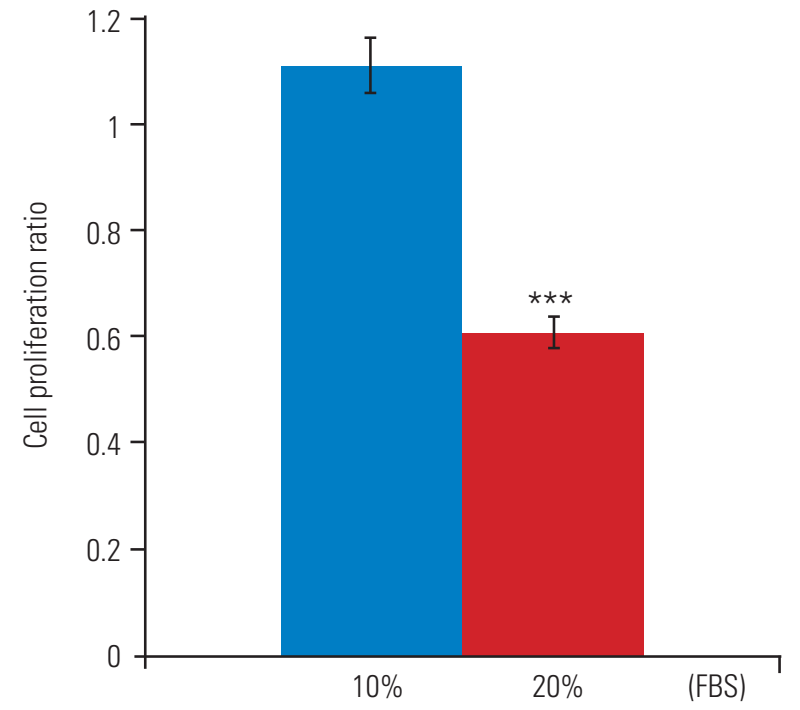

Fig. 2. MTS proliferation assay showing $45.3 \%$ reduction in proliferation of disc cells incubated in $0 \%$ fetal bovine serum (FBS) compared to those incubated in $10 \%$ FBS $(0.608 \pm 0.12$ vs. $1.111 \pm 0.24)$. MTS, 3-(4,5-dimethylthiazol-2-yl)-5-(3-carboxymethoxyphenyl)-2-(4sulfophenyl)-2H-tetrazolium. ${ }^{* * *} p<0.001$.

was significantly lower than that of disc cells cultured in $10 \% \mathrm{FBS}$ after 48 hours $(0.608 \pm 0.12$ vs. $1.111 \pm 0.24$, $p<0.001$ ) (Fig. 2). Furthermore, Western blot analysis demonstrated that Fas expression was upregulated in serum-deprived disc cells compared to those incubated under normal serum conditions (Fig. 3). These results strongly suggested that the upregulation of Fas expression was responsible for the increased apoptosis and decreased proliferation of disc cells induced by serum deprivation. 


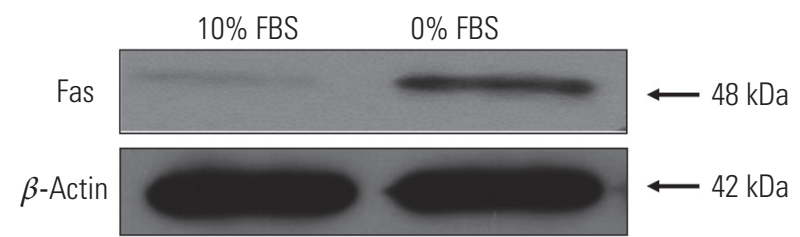

Fig. 3. Western blot analysis demonstrating that Fas expression was upregulated in disc cells incubated in $0 \%$ fetal bovine serum (FBS) compared to those cultured in 10\% FBS.
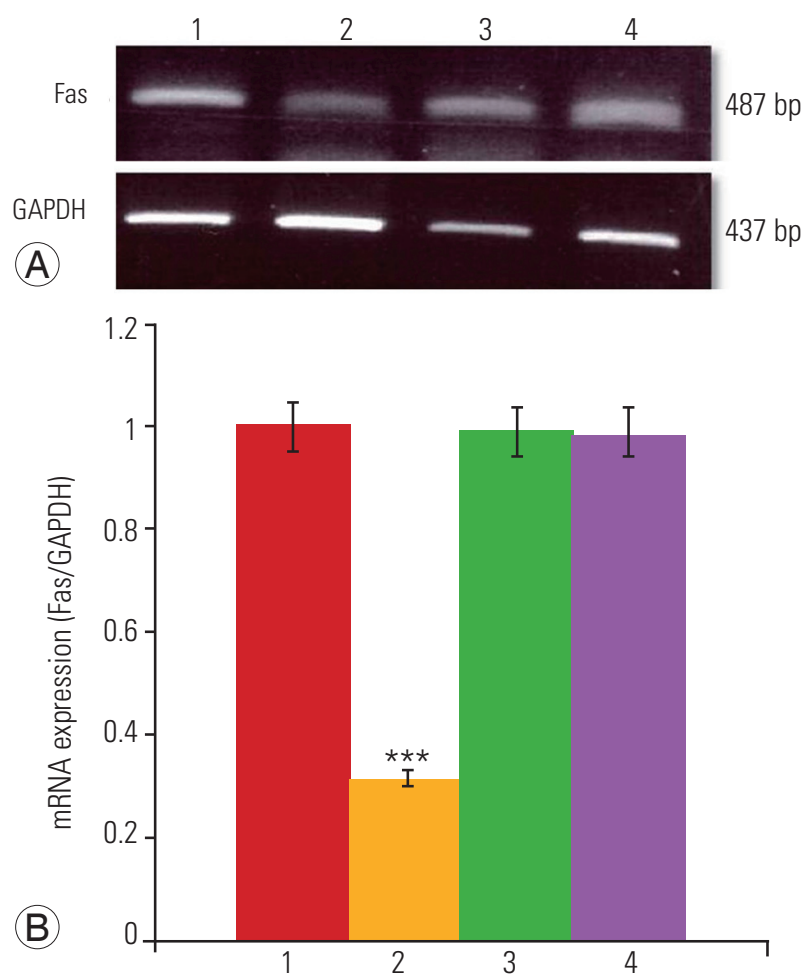

Fig. 4. (A) Analysis of Fas levels by reverse transcription-polymerase chain reaction. Fas siRNA significantly suppressed Fas mRNA levels in disc cells cultured in $0 \%$ fetal bovine serum (FBS) compared to those transfected with negative siRNA or the transfection agent alone (Mock). Fas mRNA was reduced by $68.5 \%$ with Fas siRNA ( $p<0.001)$.

(B) Note that Fas mRNA levels did not change in negative siRNAor Mock-transfected cultures under conditions of serum deprivation. siRNA, small interfering RNA; 1, 0\% FBS; 2, 0\% FBS+Fas siRNA; 3, 0\% FBS+negative siRNA; 4, 0\% FBS+Mock. GAPDH, glyceraldehyde-3phosphate dehydrogenase. ${ }^{* *} p<0.001$.

\section{2. siRNA-mediated suppression of Fas in disc cells}

The results of RT-PCR analysis are presented in Fig. 4. As shown in Fig. 4A, Fas siRNA significantly suppressed Fas mRNA levels in disc cells cultured in 0\% FBS compared to those transfected with negative siRNA or the transfection reagent alone (Mock). Quantification showed that

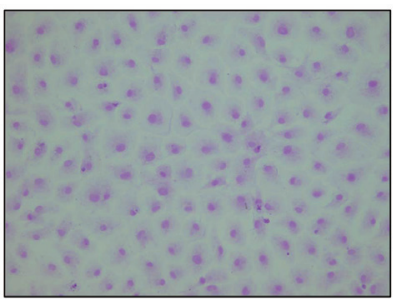

$0 \%$ FBS

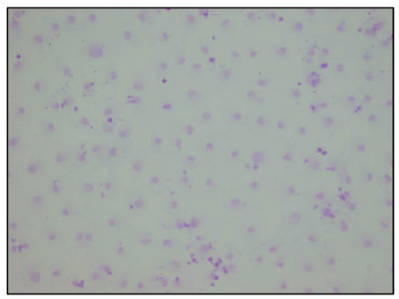

0\% FBS+Negative siRNA

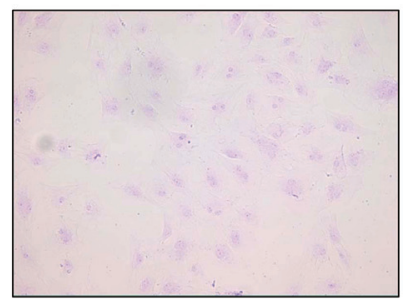

$0 \%$ FBS+Fas siRNA

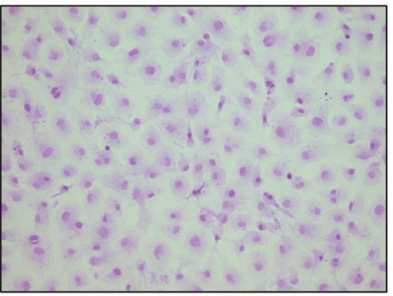

$0 \%$ FBS+Mock
Fig. 5. Terminal deoxynucleotidyl transferase-mediated dUTP nick-end labeling assay (x400) demonstrating significantly decreased apoptosis of Fas small interfering RNA (siRNA) -transfected disc cells compared to negative siRNA- and Mock-transfected disc cells in conditions of serum deprivation. FBS, fetal bovine serum.

there was a $68.5 \%$ reduction in Fas mRNA in serumdeprived Fas siRNA-transfected disc cells $(p<0.001)$ (Fig. $4 \mathrm{~B})$. Conversely, neither negative siRNA nor Mock led to a reduction in Fas mRNA levels in cells cultured under conditions of serum deprivation.

\section{Effect of siRNA-mediated Fas suppression on apopto- sis and proliferation of disc cells}

The TUNEL assay demonstrated that under conditions of serum deprivation, Fas siRNA significantly decreased apoptotic death of disc cells compared to those transfected with negative siRNA or Mock-transfected cells (Fig. 5). As presented in Fig. 6, flow cytometry analysis revealed that Fas siRNA significantly inhibited apoptosis by $9.3 \%$ in cells cultured in $0 \%$ FBS $(36.9 \% \pm 2.7 \%$ vs. $46.2 \% \pm 3.5 \%$, $p<0.05)$. In contrast, neither negative siRNA nor Mock-inhibited serum deprivation-induced apoptosis of disc cells. Finally, Fas siRNA led to a significant increase of $21 \%$ in proliferation in cells cultured in $0 \%$ FBS $(0.736 \pm 0.17$ vs. $0.608 \pm 0.12, p<0.05)$, whereas increased proliferation was not observed in serum-deprived cultures transfected with Mock-transfected or negative siRNA (Fig. 7).

\section{Discussion}

Selective silencing of endogenous genes by siRNA is uti- 

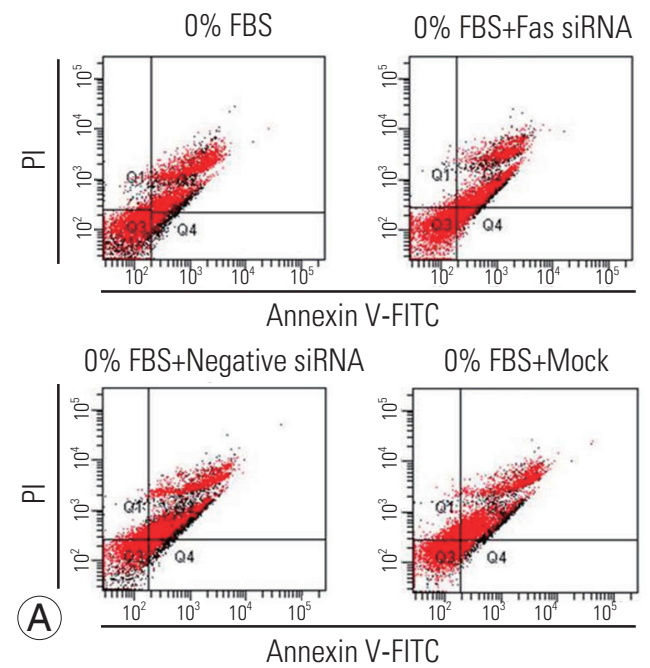

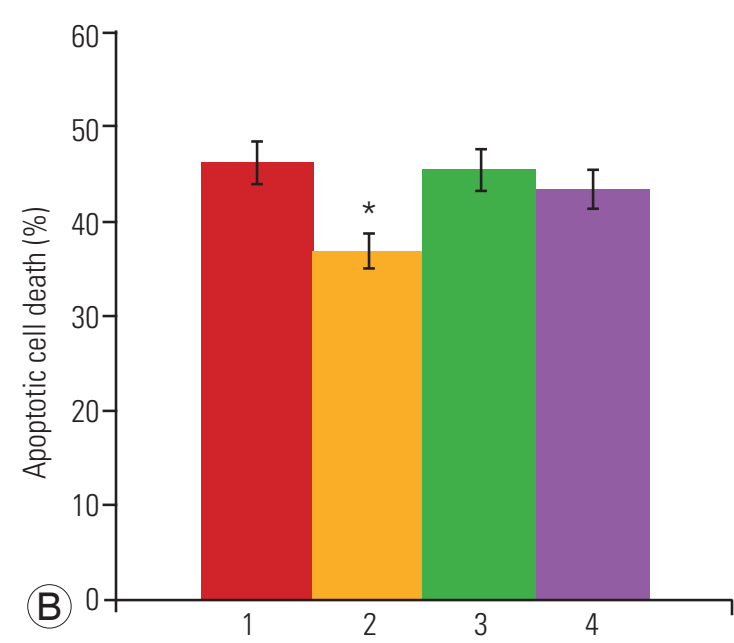

Fig. 6. (A) Flow cytometric analysis. Fas siRNA reduced apoptosis by $9.3 \%$ in disc cells cultured in $0 \%$ fetal bovine serum (FBS) $(36.9 \% \pm 2.7 \%$ vs. $46.2 \% \pm 3.5 \%)$. Pl, propidium iodide; V-FITC, Fluorescein isothiocyanate-conjugated Annexin V. (B) Note that the rate of apoptotic death of cell cultures in $0 \%$ FBS was not inhibited in negative siRNA- or Mock-transfected cultures. siRNA, small interfering RNA; siRNA, small interfering RNA; 1, 0\% FBS; 2, 0\% FBS+Fas siRNA; 3, 0\% FBS+negative siRNA; 4 , $0 \%$ FBS+Mock. * $p<0.05$.

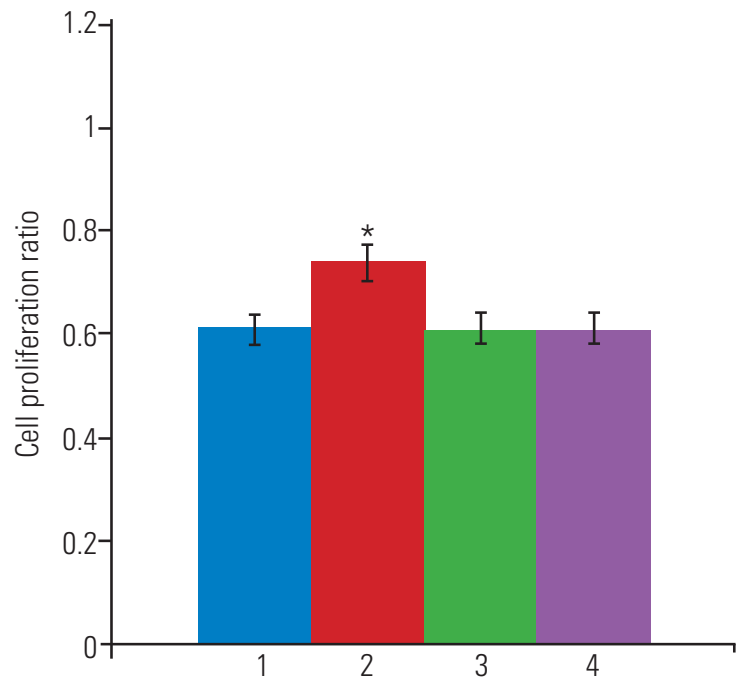

Fig. 7. MTS proliferation assay showing that Fas siRNA significantly increased proliferation by $21 \%$ in disc cells cultured in $0 \%$ fetal bovine serum (FBS) $(0.736 \pm 0.17$ vs. $0.608 \pm 0.12)$. Note that the rate of proliferation of cells cultured in $0 \%$ FBS was not increased in negative siRNAor Mock-transfected cells. siRNA, small interfering RNA; 1, 0\% FBS; 2, 0\% FBS+Fas siRNA; 3, 0\% FBS+negative siRNA; 4, 0\% FBS+Mock; MTS, 3-(4,5-dimethylthiazol-2-yl)-5-(3-carboxymethoxyphenyl)-2-(4sulfophenyl)-2H-tetrazolium. ${ }^{*} p<0.05$.

lized extensively [1-4]. Among numerous advantages of this technology are the relative ease of synthetic siRNA construction and application. Moreover, siRNA is efficient: a single dose of siRNA can sustain RNAi sufficiently long to allow recovery of cellular regulatory systems $[3,4]$. In the current study, we demonstrated that serum depri- vation increased apoptosis and decreased proliferation of disc cells, with upregulation of Fas. These results strongly suggested that Fas upregulation was responsible for the increased apoptosis and decreased proliferation of disc cells, resulting in disc degeneration. Therefore, we believe that specific downregulation of Fas by siRNA is a potential therapeutic approach to disc degeneration due to inappropriate apoptosis and proliferation of disc cells. To that end, we utilized siRNA oligonucleotides targeting Fas to transfect into disc cells for 48 hours under conditions of serum deprivation. Fas siRNA inhibited Fas expression in disc cells by approximately $68.5 \%$ at the mRNA level. We believe that the inhibitory effect of siRNA on Fas was more rapid and effective than expected, as complete serum deprivation inhibited cell survival. Therefore, our results suggested that siRNA silencing of Fas should facilitate the investigation of apoptotic pathways in disc cells in models of disc degeneration. According to the literature review, this is the first study to demonstrate siRNA-mediated suppression of Fas expression in disc cells.

In studies to characterize biologic repair of degenerated discs, several anti-apoptotic agents were shown to attenuate or prevent apoptosis of disc cells under various experimental conditions [11-14]. Growth factors such as platelet-derived growth factor, insulin-like growth factor-1, and nerve growth factor exert prosurvival, antiapoptotic effects on disc cells $[15,16]$. However, the range of anti-apoptotic effects of growth factors varies from $0.5 \%$ 
to $5 \%$ depending on the experimental conditions and dosages. Although cocktail therapy with growth factors was shown to induce the anti-apoptotic effect by $9 \%$ [16], the optimal dosage of individual growth factors for reducing apoptosis of disc cells is at present unclear. Moreover, the specific mechanisms and/or downstream pathways related to the anti-apoptotic effect of each growth factor should be investigated.

Apoptosis is mediated by the activation of caspases. Caspases act as either initiators (caspase 8 or caspase 9) or a common executioner (caspase 3 ) of apoptosis. Therefore, an alternative strategy involves interference with caspase activation. Previous studies reported that caspase inhibitors attenuated apoptosis of disc cells $[17,18]$. However, caspase inhibitors block apoptosis after its initiation or at a later stage. Therefore, targeting caspase activation may not be effective, as activation of early apoptotic signals might have detrimental effects on disc cell metabolism and activity. Moreover, even in the same cell type, caspase activation differs depending on the apoptotic stimulus. These limitations provide further support for the utilization of siRNA technology to target early stages of apoptosis and to act prior to caspase activation.

Finally, we investigated the effect of siRNA-mediated Fas suppression on the apoptosis and proliferation of disc cells under conditions of serum deprivation. The efficacy of therapeutic apoptosis inhibition by Fas siRNA (approximately $10 \%$ ) was superior to that reported for nerve growth factor and insulin-like growth factor 1 ( $2 \%$ and $5 \%$, respectively) [15]. In addition, siRNA-mediated suppression of Fas expression significantly increased cell proliferation by approximately $21 \%$. These results suggested that downregulation of Fas expression during the initiation stage of apoptosis can attenuate or delay the onset of apoptosis and induce proliferation, leading to enhanced viability of disc cells. Therefore, we believe that this dual positive effect of Fas siRNA is a powerful therapeutic approach for disc degeneration.

There were several limitations to the current study. Percentage of cell death was used as the primary outcome measure; however, assessment of cell metabolism and activity as well as the ability to produce extracellular matrix remain critical measures. For biologic repair of degenerated discs, survival and proliferation of disc cells must be followed by a corresponding increase in extracellular matrix synthesis. Disc cell survival and proliferation without positive anabolic function does not truly lead to the re- generation of a degenerated disc. Therefore, extracellular matrix synthesis should be investigated to maximize the efficacy of therapeutic siRNA targeting Fas in disc cells. Finally, while Fas siRNA led to more than $50 \%$ reduction in Fas expression, comparable changes in apoptosis and proliferation were not observed. Therefore, the efficacy of Fas siRNA on disc cell viability should be enhanced for robust regeneration of degenerated discs.

\section{Conclusions}

The observed dual positive effect of Fas siRNA might be a powerful therapeutic approach for disc degeneration by suppression of harmful gene expression. Furthermore, these findings suggest that siRNA can be used in gene function studies on disc degeneration.

\section{Conflict of Interest}

No potential conflict of interest relevant to this article was reported.

\section{References}

1. Miao GY, Lu QM, Zhang XL. Downregulation of survivin by RNAi inhibits growth of human gastric carcinoma cells. World J Gastroenterol 2007;13:11704.

2. Rossi A, Ciafre S, Balsamo M, Pierimarchi P, Santoro MG. Targeting the heat shock factor 1 by RNA interference: a potent tool to enhance hyperthermochemotherapy efficacy in cervical cancer. Cancer Res 2006;66:7678-85.

3. Tuschl T, Borkhardt A. Small interfering RNAs: a revolutionary tool for the analysis of gene function and gene therapy. Mol Interv 2002;2:158-67.

4. Zender L, Kubicka S. SiRNA based strategies for inhibition of apoptotic pathways in vivo: analytical and therapeutic implications. Apoptosis 2004;9:51-4.

5. Krammer PH. CD95(APO-1/Fas)-mediated apoptosis: live and let die. Adv Immunol 1999;71:163-210.

6. Nagata S. Apoptosis by death factor. Cell 1997;88:35565.

7. Park JB, Kim KW, Han CW, Chang H. Expression of Fas receptor on disc cells in herniated lumbar disc tissue. Spine (Phila Pa 1976) 2001;26:142-6.

8. Park JB, Chang H, Kim KW. Expression of Fas ligand 
and apoptosis of disc cells in herniated lumbar disc tissue. Spine (Phila Pa 1976) 2001;26:618-21.

9. Park JB, Lee JK, Park SJ, Kim KW, Riew KD. Mitochondrial involvement in fas-mediated apoptosis of human lumbar disc cells. J Bone Joint Surg Am 2005;87:1338-42.

10. Park JB, Chang DG, Oh SY, Park EY. Effect of RNA interference-mediated suppression of p75 on the viability of rat notochordal cells. Asian Spine J 2016;10:985-92.

11. Masuda K. Biological repair of the degenerated intervertebral disc by the injection of growth factors. Eur Spine J 2008;17 Suppl 4:441-51.

12. Yoon ST, Patel NM. Molecular therapy of the intervertebral disc. Eur Spine J 2006;15 Suppl 3:S379-88.

13. Zhao CQ, Jiang LS, Dai LY. Programmed cell death in intervertebral disc degeneration. Apoptosis 2006;11:2079-88.

14. Choi YS. Pathophysiology of degenerative disc dis- ease. Asian Spine J 2009;3:39-44.

15. Gruber HE, Norton HJ, Hanley EN Jr. Anti-apoptotic effects of IGF-1 and PDGF on human intervertebral disc cells in vitro. Spine (Phila $\mathrm{Pa} 1976$ ) 2000;25:2153-7.

16. Park JB, Kim YB, Park EY. Synergistic effect of nerve growth factor and insulin-like growth factor-1 on providing a pro-survival, anti-apoptotic benefit and increased extracellular matrix synthesis in stressed rat intervertebral disc cells. J Neurol Sci Turk 2015; 32:728-37.

17. Kim KW, Ha KY, Lee JS, Rhyu KW, An HS, Woo YK. The apoptotic effects of oxidative stress and antiapoptotic effects of caspase inhibitors on rat notochordal cells. Spine (Phila Pa 1976) 2007;32:2443-8.

18. Park JB, Park IC, Park SJ, Jin HO, Lee JK, Riew KD. Anti-apoptotic effects of caspase inhibitors on rat intervertebral disc cells. J Bone Joint Surg Am 2006;88:771-9. 